\title{
LA CONTABILIDAD Y SU DESAFIO ANTE EL DESARROLLO SUSTENTABLE
}

\section{ACCOUNTING AND ITS CHALLENGE TO SUSTAINABLE DEVELOPMENT}

\author{
Saulo Sinforoso Martínez*, Jerónimo Domingo Ricárdez Jiménez **, \\ Natalia Murrieta Martínez ${ }^{\star \star \star}$
}

\begin{abstract}
*Maestría en Ciencias Administrativas. Docente adscrito a la Facultad de Contaduría y Administración de la Universidad Veracruzana, Xalapa.

**Doctor en Ciencias Económicas. Director de la Facultad de Contaduría y Administración de la Universidad Veracruzana, Xalapa. jricardez@uv.mx

***Maestría en Contabilidad e Impuesto. Secretaria Académica de la Facultad de Contaduría y Administración de la Universidad Veracruzana, Xalapa.nmurrieta@uv.mx
\end{abstract}

Dirección para recibir correspondencia: ssinforoso@uv.mx

Fecha de recibido: 12 de julio de 2017

Fecha de aceptación: 20 de septiembre de 2017 


\section{LA CONTABILIDAD Y SU DESAFIO ANTE EL DESARROLLO SUSTENTABLE}

\section{RESUMEN}

OBJETIVO: Analizar el desarrollo sustentable desde la perspectiva contable y su impacto en un programa educativo del área Económico Administrativa de la Universidad Veracruzana

MATERIAL Y MÉTODO: Se inicia con las aportaciones de autores como Gray, Larrinaga, Llull, Pelegrín, entre otros, se continúa con las corrientes teóricas que sustentan la vinculación entre la Contabilidad y el desarrollo sustentable, analizando un estudio de caso. Esta investigación es con enfoque mixto con alcance descriptivo bajo un estudio de caso.

RESULTADOS: Los resultados muestran que el desarrollo sustentable desde la perspectiva de la Contabilidad se ha convertido en una línea de investigación, el programa educativo de Licenciado en Contaduría contiene algunos temas vinculados con el desarrollo sustentable, sin embargo presenta carencias y limitantes.

CONCLUSIÓN: La Contabilidad ha dejado de ser una disciplina enfocada al registro y presentación de la información contable y ha evolucionado a una ciencia que incursiona en el ámbito de la investigación teniendo como una de sus líneas de estudio el desarrollo sustentable, por tal motivo las Instituciones de Educación Superior deben incluir en sus saberes teóricos esta temática

PALABRAS CLAVE: Contabilidad. Desarrollo sustentable. Universidad Veracruzana. Programa educativo. Licenciado en Contaduría. 


\section{LA CONTABILIDAD Y SU DESAFIO ANTE EL DESARROLLO SUSTENTABLE}

\section{ABSTRACT}

OBJECTIVE: To Analyze sustainable development from the accounting perspective and its impact on an educational program of the Economic-Administrative Area of the Universidad Veracruzana.

MATERIAL AND METHOD: The research begins with the contributions of authors such as Gray, Larrinaga, Llull, Pelegrin, among others. Then, it continues with the theoretical currents that support the link between Accounting and sustainable development, analyzing a case study. This research has a mixed approach with a descriptive scope related to a case study.

RESULTS: The results show that sustainable development from the perspective of Accounting has become a line of research, the educational program of bachelor's degree in Accounting contains some topics related to sustainable development, however it has shortcomings and limitations.

CONCLUSIÓN: Accounting has ceased to be a discipline focused on the recording and presentation of accounting information and has evolved into a science that dabbles in the field of research having as one of its study lines the sustainable development, for this reason, the Institutions of Higher Education must include in their theoretical knowledge this topic.

KEYWORDS: Accounting. Sustainable development. Universidad Veracruzana. Educational program. Bachelor's degree in accounting. 


\section{LA CONTABILIDAD Y SU DESAFIO ANTE EL DESARROLLO SUSTENTABLE}

\section{MARCO TEÓRICO}

El desarrollo sustentable tiene sus inicios en 1972 y en el año de 1992 retoma fuerza al realizarse la Cumbre de la Tierra en Río de Janeiro, participando 178 países, englobando temas sociales, ambientales y económicos. El desarrollo sustentable es una línea de investigación que ha impactado en diversas disciplinas como la Contabilidad surgiendo lo que se conoce como Contabilidad Medioambiental "La parte de la Contabilidad aplicada cuyo objeto son las relaciones entre una entidad y su medio ambiente "(Fernández, 2004, p. 33), clasificándola según Llena (1999) en Nacional, Financiera, de Gestión y de Dirección Estratégica.

Hablar de Contabilidad Nacional abarca experiencias educativas como Economía, si se trata de Contabilidad Financiera Medioambiental engloba a Fundamentos de Contabilidad, Contabilidad Intermedia I y II, entre otras. En relación a la Gestión la Contabilidad de Costo y cuando se enfoca a la Dirección Estratégica comprende la Contabilidad Administrativa.

\section{Enfoques teóricos de la Contabilidad Ambiental}

En la tabla 1, se muestran las posturas teóricas que sustentan el estudio del desarrollo sustentable desde la Contabilidad.

\section{Tabla 1}

Posturas teóricas entre la Contabilidad y el desarrollo sustentable

I. Teoría Neoclásica o Marginalista

II. Paradigma del Beneficio Verdadero

III. Corriente Crítica Radical

IV. Teoría de Utilidad para la Toma de Decisiones

V. Teoría de Legitimación

VI. Teoría de los Stakeholders

VII. Teoría del Accountability

Fuente: Elaboración propia. 


\section{LA CONTABILIDAD Y SU DESAFIO ANTE EL DESARROLLO SUSTENTABLE}

\section{Teoría Neoclásica o Marginalista}

De acuerdo a Sastre:

"La mayor parte de las teorías contables tradicionales se desarrollan en este contexto, asumiendo por lo tanto que la maximización del interés individual a través del mercado dará lugar a la maximización de la eficiencia económica y ello a su vez al bienestar de la sociedad en su conjunto" (1997, p. 4).

Carmona, Carrasco y Fernández (1993) afirman que la información de las implicaciones ambientales o sociales ocasionados por la empresa, debe construirse de datos duros, datos medioambientales, cuantitativos o/y cualitativos y datos sociales, pues es parte de la función de la Contabilidad el reflejar la imagen de la empresas a través de la información.

\section{Paradigma del beneficio verdadero}

Este paradigma "Sostiene que la empresa en la determinación de sus resultados ignora los efectos sociales y medioambientales por lo que, la información dada a través de la Contabilidad social podría medir su contribución neta a la sociedad" (Díaz, 2003, p.33). Además "Se orienta a la medición en abstracto de hechos pasados, en búsqueda de un concepto único y autosuficiente de verdad económica" (Tua, 1995, p. 191). Desde esta postura Parada y Perdomo (2014, p. 9), Ullmann (1976), Ramanathan (1976) y Estes (1972) señalan que si las organizaciones reconocen contablemente los impactos sociales y ambientales se obtendría una información contable completa que ayudaría a los tomadores de decisiones a contribuir al cuidado y conservación del medio ambiente pues se tomarían decisiones certeras.

\section{Corriente crítica radical}

Impulsada por Burchell et al., 1980; Tinker, 1985; Lehman, 1995; Bell y Lehman, 1999 y Puxty, 1993. Para Llull (2001, p.111) estos autores "Parten de la existencia de intereses enfrentados entre los diferentes grupos sociales interesados en la empresa, y consideran a la Contabilidad como una institución legitimadora de la actuación de la empresa en todos los aspectos, incluidos los sociales y medioambientales". 


\section{LA CONTABILIDAD Y SU DESAFIO ANTE EL DESARROLLO SUSTENTABLE}

\section{Teoría de utilidad para la toma de decisiones}

Parada y Perdomo (2014) consideran pertinente la Teoría de la Utilidad para los estudios ambientales desde la postura contable pues argumentan que "Las empresas deben informar a la sociedad sobre aquello que la afecta, con el fin de que sea útil para la toma de decisiones" (2014, p. 6). Tua (1995, p. 191) afirma que bajo esta teoría "El contenido de los estados financieros se orienta a cubrir las necesidades de los usuarios; constituye por lo tanto la información un soporte vital para la adecuada toma de decisiones".

\section{Teoría de legitimación}

Algunos autores (Guthrie y Parker, 1989; O`dwyer, 2002; Deegan, 2002; Husillos, 2004) en Gómez (2009, p.75) la consideran como "El proceso organizacional de presentarse como una entidad alineada con los valores e instituciones sociales dominantes, para justificar su existencia y presencia en la sociedad". Se entiende por legitimidad "Una condición o estatus que emana de la congruencia entre el sistema de valores de una corporación y el de la sociedad de la que forma parte" (Husillo, 2004, p.100).

\section{Teoría de los stakeholders}

Llena (2008, p.126) señala que "Bajo la perspectiva de la teoría del Stakeholder, la Contabilidad y la profesión contable debe colaborar en el cumplimiento de las necesidades de los agentes interesados ofreciendo información y colaborando en la gestión medioambiental por medio de diversas funciones", postura que defienden Gray, Owen y Adams (1996), mientras que Carrasco, Correa y Larrinaga (1999) indican que esta teoría no se enfoca en la información completa si no que los partícipes se involucren en el tema del medio ambiente.

\section{Teoría del accountability}

Bautizada por Gray, Owen y Adams (1996) esta teoría se confunde con la teoría anterior; sin embargo, Llull (2001) afirma que la diferencia recae en que la teoría de los Stakeholders sólo se incluye grupos con intereses directos en la empresa, excluyendo a la sociedad como un todo. Williams (1987, p. 170) define el Accountability como "Una relación obligatoria creada mediante las transacciones en las que se espera que una parte de cuenta de sus acciones a otras partes". 


\section{LA CONTABILIDAD Y SU DESAFIO ANTE EL DESARROLLO SUSTENTABLE}

\section{Impacto del desarrollo sustentable en la profesión contable}

La Organización de las Naciones Unidas para la Educación, la Ciencia y la Cultura (UNESCO) afirma que:

"El desarrollo sostenible no se logra únicamente con soluciones tecnológicas, medidas políticas y recursos económicos: también debemos cambiar nuestra forma de pensar y actuar. Para conseguirlo, se necesita una educación para el desarrollo sostenible de calidad a todos los niveles y en todos los contextos sociales".

En ese sentido, se recomienda abordar el tema de desarrollo sustentable desde la Instituciones de Educación Superior pues "La universidad como entidad docente e investigadora, debería ser el principal agente de cambio que proporcionara respuestas a los problemas y a los retos de la sociedad actual" (Gutiérrez, Benayas \& Calvo, 2006, 56), por tal motivo surge la necesidad de diagnosticar en qué grado las Instituciones de Educación Superior han agregado en sus programas de estudios temas vinculados con el desarrollo sustentable, pues los programas educativos ambientales, dentro del paradigma del desarrollo sustentable, se convierte para la comunidades en una alternativa en la resolución de sus problemas ambientales, tanto en el ámbito ecológico como en el económico, social y cultural "(Zambrano, Castillo y Sánchez, 2011. P. 82).

\section{MATERIAL Y MÉTODO}

Este estudio se aborda con un enfoque mixto con alcance descriptivo bajo un estudio de caso. Se inicia con una revisión teórica y aportes de diversos autores que fundamentan la vinculación entre la Contabilidad y el desarrollo sustentable. Se realiza un análisis de todos los programas de estudio del programa educativo de Licenciado en Contaduría de la Universidad Veracruzana y se identifica qué saberes se han incluido para estudiar los temas de desarrollo sustentable en los estudiantes.

\section{RESULTADOS}

En la tabla 2, se muestran los resultados del análisis del mapa curricular del programa educativo sujeto de estudio. 


\section{LA CONTABILIDAD Y SU DESAFIO ANTE EL DESARROLLO SUSTENTABLE}

\section{Tabla 2}

Mapa curricular programa educativo Licenciado en Contaduría de la Universidad Veracruzana

\begin{tabular}{lcc}
\hline & Frecuencia absoluta & Frecuencia relativa \\
\hline $\begin{array}{l}\text { Experiencias educativas que } \\
\text { abordan temas de desarrollo }\end{array}$ & 8 & $17 \%$ \\
sustentable & & \\
$\begin{array}{l}\text { Experiencias educativas que NO } \\
\text { abordan temas de desarrollo }\end{array}$ & 39 & $83 \%$ \\
sustentable & & \\
Total experiencias educativas & 47 & $100 \%$ \\
analizadas & & \\
\hline
\end{tabular}

Fuente: Elaboración propia con datos de la Universidad Veracruzana, 2017.

El $83 \%$ de las experiencias educativas pertenecientes al mapa curricular del programa educativo de Licenciado en Contaduría de la Universidad Veracruzana no integran el tema de desarrollo sustentable (ver tabla 2), mientras que el $17 \%$ si lo incluye como parte de sus temáticas esto se aprecia en la figura1.

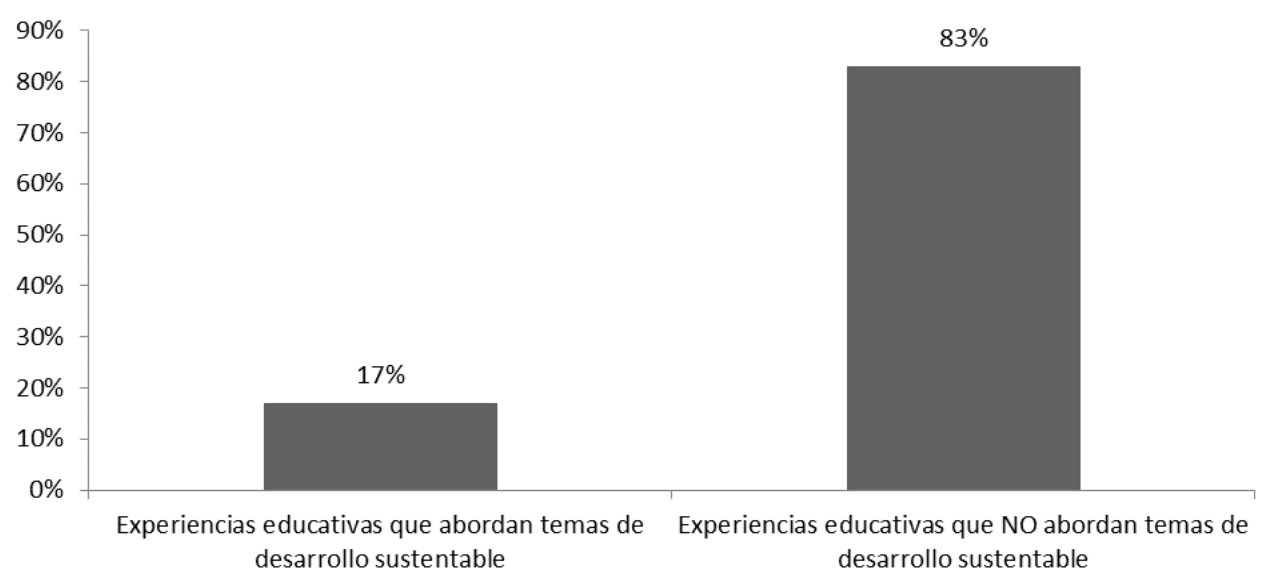

Figura 1. Mapa curricular programa educativo Licenciado en Contaduría.

Fuente: Elaboración propia con datos de la Universidad Veracruzana, 2017. 


\section{LA CONTABILIDAD Y SU DESAFIO ANTE EL DESARROLLO SUSTENTABLE}

\section{Tabla 3}

Forma cuantitativa y cualitativa de las experiencias educativas que comprenden temas de desarrollo sustentable por número de temas

\begin{tabular}{|c|c|c|c|}
\hline Experiencias educativas & Temas & $\begin{array}{l}\text { Frecuencia } \\
\text { absoluta }\end{array}$ & $\begin{array}{l}\text { Frecuencia } \\
\text { Relativa }\end{array}$ \\
\hline Economía & $\begin{array}{l}\text { Desarrollo sustentable, sostenible o } \\
\text { perdurable. }\end{array}$ & 1 & $6.67 \%$ \\
\hline $\begin{array}{l}\text { Fundamentos de la } \\
\text { administración }\end{array}$ & $\begin{aligned} &> \text { Las dimensiones del desarrollo } \\
& \text { sustentable } \\
&>\text { Educación ambiental, redes de } \\
& \text { educación ambiental y desarrollo } \\
& \text { sustentable } \\
&>\text { Ecoturismo y desarrollo sustentable } \\
&>\text { Enfoque tecnológico del desarrollo } \\
& \text { sustentable. } \\
&>\text { Enfoque económico y normativo del } \\
& \text { desarrollo sustentable. }\end{aligned}$ & 5 & $33.33 \%$ \\
\hline $\begin{array}{l}\text { Desarrollo de } \\
\text { emprendedores }\end{array}$ & $\begin{array}{l}>\text { Responsabilidad Social Empresarial } \\
\text { Programas de beneficio a los } \\
\text { Stakeholders. } \\
\text { Programas de protección y conservación } \\
\text { del medio ambiente }\end{array}$ & 3 & $20 \%$ \\
\hline Auditoria II & $>$ Auditoría Ambiental & 1 & $6.67 \%$ \\
\hline Contabilidad intermedia I & $\begin{array}{l}\text { Provisiones, contingencias y } \\
\text { compromisos. }\end{array}$ & 1 & $6.67 \%$ \\
\hline Contabilidad intermedia II & $>$ Activos intangibles & 1 & $6.67 \%$ \\
\hline Proyectos de inversión & $\begin{array}{l}\text { Evaluación social y de sustentabilidad } \\
\text { del proyecto. } \\
>\text { Consideración de los posibles impactos } \\
\text { Social y Ecológico. }\end{array}$ & 2 & $13.33 \%$ \\
\hline $\begin{array}{l}\text { Mercados e instituciones } \\
\text { financieras }\end{array}$ & $\begin{array}{l}\text { Gestión de riesgos ambientales } \\
\text { mediante productos financieros } \\
\text { derivados. }\end{array}$ & 1 & $6.67 \%$ \\
\hline Total & & 15 & $100 \%$ \\
\hline
\end{tabular}

Fuente: Elaboración propia con datos de la Universidad Veracruzana, 2017.

La experiencia educativa que incluye la mayor cantidad de contenidos enfocados al desarrollo sustentable es la de Fundamentos de la Administración con el 33.33\% esto es equivalente a cinco temas de este programa de estudio destinado al desarrollo sustentable ( Ver tabla 3), continuando con Desarrollo de Emprendedores con un 20\% y en menor medida las 


\section{LA CONTABILIDAD Y SU DESAFIO ANTE EL DESARROLLO SUSTENTABLE}

experiencias educativas de Mercados e Instituciones Financieras, Contabilidad intermedia I y II, Auditoria II y Economía ( ver figura 2).

\section{Figura 2}

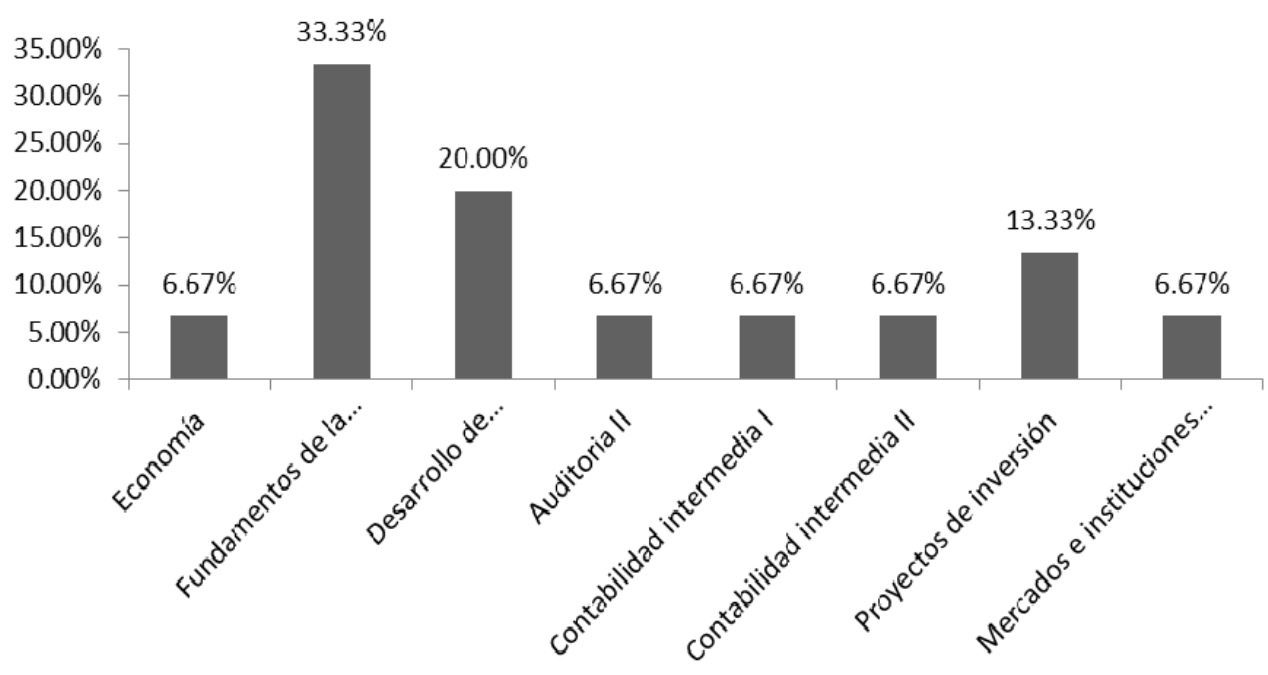

luría de la

Universidad Veracruzana vinculada con el desarrollo sustentable por número de temas.

Fuente: Elaboración propia con datos de la Universidad Veracruzana, 2017.

\section{Tabla 4}

Experiencias educativas del programa educativo Licenciado en Contaduría de la Universidad Veracruzana vinculada con el desarrollo sustentable por área disciplinar

\begin{tabular}{|c|c|c|c|}
\hline Área disciplinar & Experiencias educativas & $\begin{array}{l}\text { Frecuencia } \\
\text { absoluta }\end{array}$ & $\begin{array}{l}\text { Frecuencia } \\
\text { Relativa }\end{array}$ \\
\hline Área de formación básica & - & 0 & $0 \%$ \\
\hline Iniciación a la disciplina & $\begin{array}{l}\text { Economía } \\
>\text { Fundamentos de la } \\
\text { administración }\end{array}$ & 2 & $25 \%$ \\
\hline Área de formación disciplinaria & $\begin{array}{l}>\text { Desarrollo de emprendedores } \\
>\text { Auditoria II } \\
>\text { Contabilidad intermedia I } \\
>\text { Contabilidad intermedia II } \\
>\text { Proyectos de inversión } \\
>\text { Mercados e instituciones } \\
\text { financieras }\end{array}$ & 6 & $75 \%$ \\
\hline Área de formación terminal & - & 0 & $0 \%$ \\
\hline Área de elección libre & - & 0 & $0 \%$ \\
\hline Total & & 8 & $100 \%$ \\
\hline
\end{tabular}

Fuente: Elaboración propia con datos de la Universidad Veracruzana, 2017. 


\section{LA CONTABILIDAD Y SU DESAFIO ANTE EL DESARROLLO SUSTENTABLE}

El $75 \%$ de las experiencias educativas que comprenden temas de desarrollo sustentable pertenecen al área de formación disciplinar (Ver tabla 4), mientras que el 25\% a la iniciación a la disciplina. El área de formación básica, de formación terminal y de elección libre no incluyen temas de desarrollo sustentable (ver figura 3).

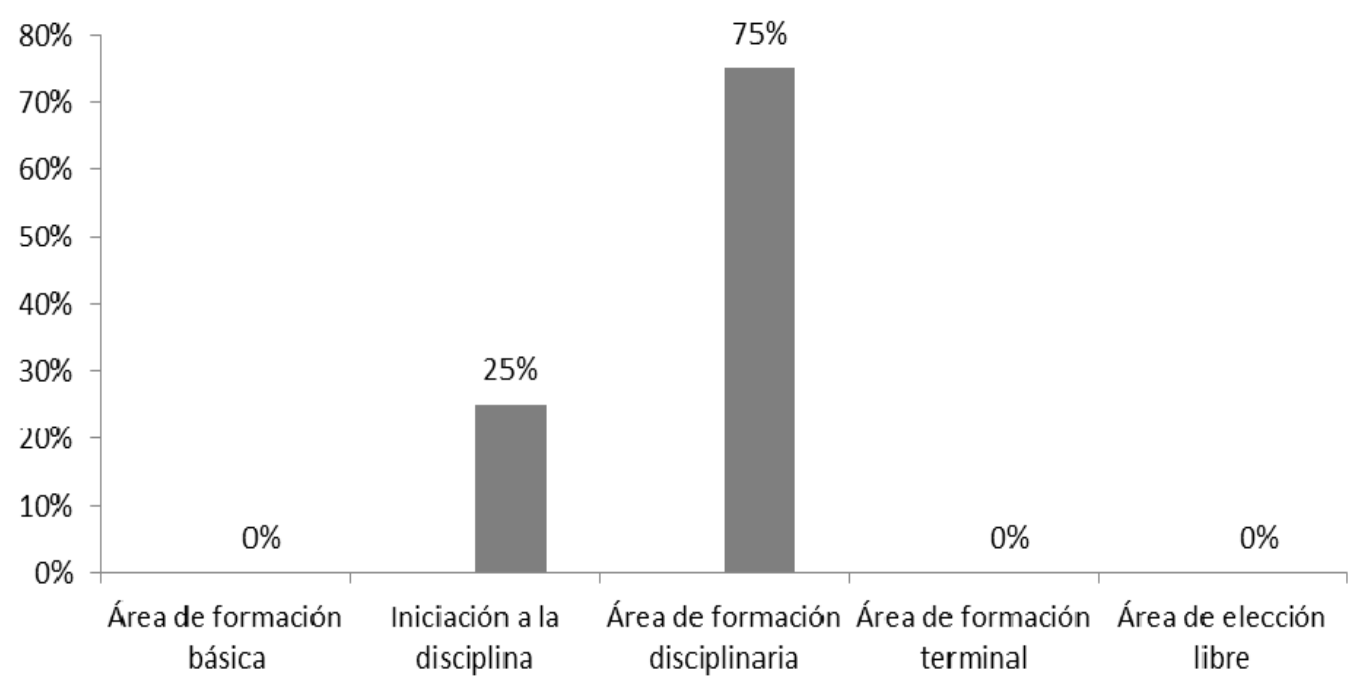

Figura 3. Experiencias educativas del programa educativo Licenciado en Contaduría de la Universidad Veracruzana vinculada con el desarrollo sustentable por Área disciplinar.

Fuente: Elaboración propia con datos de la Universidad Veracruzana, 2017.

\section{CONCLUSIÓN}

La Contabilidad dejó de ser una disciplina enfocada al registro y presentación de la información financiera y evolucionó a una ciencia que incursiona en el ámbito de la investigación teniendo como una de sus líneas de estudio el desarrollo sustentable, por tal motivo es necesario que los profesionales de esta rama cuenten con las herramientas imperantes para afrontar los nuevos desafíos. Para la sociedad el desarrollo sustentable es fundamental, para ellos las Instituciones de Educación Superior lo deben incluir en sus saberes teóricos.

El programa educativo de Licenciado en Contaduría de la Universidad Veracruzana incluye en menor medida en sus programas de estudios el tema del desarrollo sustentable, no se aborda en las áreas como los impuestos y costos. Se concluye que se debe sumar esfuerzo con apoyo de los cuerpos académicos o grupos de investigación para formar profesionales capaces de afrontar los desafíos actuales que la sociedad exige. 


\section{LA CONTABILIDAD Y SU DESAFIO ANTE EL DESARROLLO SUSTENTABLE}

\section{REFERENCIAS BIBLIOGRÁFICAS}

Bell, F. \& Lehman, G. (2000). Recent trends in environmental accounting: How green are your accounts? Accounting Forum, 23(2), pp. 175-192. Recuperado de http://dx.doi.org/10.1111/1467-6303.00010.

Burchell, S.; Clubb, C.; Hopwood, A.; Hughes, J. \& Nahapiet, J. (1998). The role of accounting in organisations and society. Accounting, organizations and society, 5(1), pp.5-27 Recuperado de https://doi.org/10.1016/0361-3682 (80)90017-3.

Carrasco, F.; Correa, C. \& Larrinaga, C. (1999). Información y gestión medioambiental: una reflexión sobre los factores de motivación, proceso de desarrollo de informes e influencia de los Stakeholders en las empresas del sector eléctrico. CD-ROM $2^{\mathrm{a}}$ Reunión sobre Investigación en Contabilidad Social y Medioambiental en el Centro de investigación en Contabilidad social y medioambiental, Valencia, España.

Carmona, S.; Carrasco, F. \& Fernández, L. (1993). Un enfoque interdisciplinar de la Contabilidad del medio ambiente. Revista Española de Financiación y Contabilidad, 23(75), pp. 277-305. Recuperado de http://aeca.es/old/pub/refc/n75.html.

Deegan, C.; Ranking, M. \& Tobin, J. (2002). An examination of the corporate social and environmental disclosures of BHP from 1983-1997. A test of legitimacy theory. Accounting, Auditing and Accountability Journal, 15(3), pp. 312-343. Recuperado de http://www.emeraldinsight.com/doi/pdfplus/10.1108/09513570210435861.

Díaz, M. (2003). La Contabilidad social-origen y paradigmas. Revistas UNMSM, 10(19), pp.3142.

Recuperado

de

http://sisbib.unmsm.edu.pe/BibVirtual/Publicaciones/quipukamayoc/2003/primer/contabilid ad.html

Estes, R. W. (1972). Socio-economic accounting and external diseconomies. The Accounting Review, 47(2), pp.284-290. Recuperado de https://www.jstor.org/stable/pdf/244751.pdf.

Fernández, C. (2004). El Marco Conceptual de la Contabilidad ambiental. Una propuesta para el debate. Contabilidad y auditoría, 10(19), pp. 29-38. Recuperado de http://ojs.econ.uba.ar/ojs/index.php/Contyaudit/article/view/141/231. 


\section{LA CONTABILIDAD Y SU DESAFIO ANTE EL DESARROLLO SUSTENTABLE}

Gómez, M. (2009). Los informes contables externos y la legitimidad organizacional con el entorno: estudio de un caso en Colombia. Revista Innovar Journal, Revista de Ciencias Administrativas y Sociales, 19(34), pp. 147-166. Recuperado de http://revistas.unal.edu.co/index.php/innovar/article/view/21611/22613. DOI: 10.15446/innovar.

Gray, R. (1992). Accounting and Environmentalism: An exploration of the challenge of gently accounting for accountability, transparency and sustainability. Accounting, Organizations and Society, 17(5), pp. 399-425. Recuperado de https://doi.org/10.1016/03613682(92)90038-T.

Gray, R., Owen, D., \& Adams, C. (1996). Accounting \& Accountability: changes and challenges in corporate social and environmental reporting. London: Prentice hall.

Gray, R., Owen, D., \& Maunders, K. (1987). Corporate social reporting. Hemel Hempstead: Prentice Hall.

Guthrie, J. \& Parker, L. (1989). Corporate social reporting: a rebuttal of legitimacy theory. Accounting and Business Research, 19(76), pp. 243-352. Recuperado de http://dx.doi.org/10.1080/00014788.1989.9728863.

Gutiérrez, J., Benayas, J. \& Calvo, S. (2006) Educación para el desarrollo sostenible: evaluación de retos y oportunidades del decenio 2005-2014. Revista Iberoamericana de Educación, 40, pp.25-69. Recuperado de http://rieoei.org/rie40a01.htm.

Husillos, J. (2004). Información medioambiental, Contabilidad y teoría de la legitimación. Empresas cotizadas: 1997-1999. Documentos de Trabajo 7, Madrid: Universidad Carlos III, Departamento de Economía de Empresa.

Larrinaga, C. (1999). Perspectivas alternativas de investigación en Contabilidad: una revisión. Revista de Contabilidad, 2(3), pp. 103-132. Recuperado de https://earchivo.uc3m.es/bitstream/handle/10016/6385/db990904.pdf.

Larrinaga, C. (1997). Consideraciones en torno a la relación entre la Contabilidad y el medio ambiente. Revista Española de Financiación y Contabilidad, 26(93), pp.957-991. Recuperado de http://aeca.es/old/refc_1972-2013/1997/93-4.pdf. 


\section{LA CONTABILIDAD Y SU DESAFIO ANTE EL DESARROLLO SUSTENTABLE}

Lehman, G.A. (1995). Legitimate concern for environmental accounting. Critical Perspectives on Accounting, 6(5), pp.393-412. Recuperado de http://www.sciencedirect.com/science/article/pii/S1045235485710374. DOI: 10.1006/cpac.1995.1037.

Llena, F. (2008). El papel de la Contabilidad en la gestión medioambiental de la empresa: propuestas y evidencia empírica. Revista de Contabilidad y Dirección, 7, pp. 103-134. Recuperado de http://www.accid.org/revista/documents/rcd7_castella_103.pdf.

Llena, F. (1999). La Contabilidad en la interacción empresa medio ambiente. Su contribución a la gestión medioambiental. Tesis Doctoral. Universidad de Zaragoza, comunidad autónoma de Aragón, España.

Llull, A, (2001). Contabilidad medioambiental y desarrollo sostenible en el sector turístico. Tesis Doctoral. Universidad de las Islas Baleares.

O'dwyer, B. (2002). Conceptions of Corporate Social Responsibility: the nature of managerial capture. Accounting. Auditing and Accountability Journal, 4(16), pp. 523-557. Recuperado de https://doi.org/10.1108/09513570310492290.

Parada, W.D. \& Perdomo, D. (2014). Contabilidad Social: ¿Una verdadera alternativa? Trabajo presentado en el XIX congreso internacional de contaduría, administración e informática en la Universidad Autónoma de México, ciudad universitaria, México, D.F.

Pelegrín, A. \& Ortiz M.L. (2013). La Contabilidad financiera medioambiental. Un análisis teórico y empírico desde diferentes contextos 1. México: Editorial Universitaria.

Puxty, A. G. \& Laughlin, R. C. (1983). A rational reconstruction of the usefulness criterion. Journal of Business Finance and Accounting, 10(4), pp. 543-560. Recuperado de http://onlinelibrary.wiley.com/doi/10.1111/j.1468-

5957.1983.tb00452.x/epdf?r3_referer=wol\&tracking_action=preview_click\&show_checkout $=1 \&$ purchase_referrer=www.google.com.co\&purchase_site_license=LICENSE_DENIED.

Ramanathan, K. V. (1976). Toward a theory of corporate social accounting. The Accounting Review, 51(3), pp.516-52. Recuperado http://www.jstor.org/stable/245462. 


\section{LA CONTABILIDAD Y SU DESAFIO ANTE EL DESARROLLO SUSTENTABLE}

Salas, H. (2015). Integración de la dimensión ambiental al sistema de información financiero de empresas ubicadas en ecosistemas frágiles. Revista Universidad y Sociedad, 7(1), pp.102-109.Recuperado de http://scielo.sld.cu/pdf/rus/v7n1/rus14115.pdf.

Sastre, J.M. (1997). Hacia una teoría explicativa de la información socio-medioambiental: aplicación al sector eléctrico español mediante la técnica del análisis de contenido. CDROM de la I Reunión sobre investigación en Contabilidad Medioambiental en la Universidad Pablo de Olavide, Sevilla, España.

Tascon, M. (1997). La Contabilidad como disciplina científica. Revista contaduría y administración, 187, pp. 69-94. Recuperado de https://aprendeenlinea.udea.edu.co/revistas/index.php/cont/article/view/25127/20624.

Terreros, D. A. (2014). Gestión ambiental: una mirada desde la Contabilidad. Activos, 12(23), pp. 227-241. Recuperado de http://dx.doi.org/10.15332/s0124-5805.2014.0023.07

Tinker, A. M, (1985). Paper prophets: A social critique of accounting, 1. New York: Praegar.

Tua, J. (1995). Lecturas de teoría e investigación contable 1. Colombia: Ediciones Gráficas Ltda.

Ullmann, A. A. (1976). The corporate environmental accounting system: A management tool for fighting environmental degradation. Accounting, Organizations \& Society, 1(1), pp.71-79. Recuperado de https://doi.org/10.1016/0361-3682(76)90008-8.

Williams, P. F. (1987). The legitimate concern with fairness. Accounting, organizations and society, 12(2), pp. 169-189. Recuperado https://doi.org/10.1016/0361-3682(87)90005-5.

Zambrano, J.G. Castillo, M. \& Sánchez, A. (2011). El desarrollo sustentable y los programas educativos ambientales. Revista Universitaria Arbitrada de Investigación y Diálogo Académico. 7(1), pp. 68-65. Recuperado de http://conhisremi.iuttol.edu.ve/pdf/ARTI000116.pdf. 